\title{
Stability of Two-Dimensional Initial Boundary Value Problems Using Leap-Frog Type Schemes
}

\author{
By Saul Abarbanel* and David Gottlieb**
}

\begin{abstract}
The paper discusses stable boundary conditions for the two-dimensional Leap-Frog scheme and the Modified Leap-Frog scheme introduced by the authors in a former work.
\end{abstract}

1. Introduction. There are several ways of extending the second-order LeapFrog finite-difference scheme to two space dimensions. Consider the hyperbolic set

$$
\frac{\partial u}{\partial t}=\frac{\partial F}{\partial x}+\frac{\partial G}{\partial y}
$$

where $u, F(u)$ and $G(u)$ are $m$-component vectors. The standard way of applying the Leap-Frog scheme to (1.1) is as follows:

$$
u_{j, k}^{n+1}=u_{j, k}^{n-1}+\frac{\Delta t}{\Delta x}\left(F_{j+1, k}^{n}-F_{j-1, k}^{n}\right)+\frac{\Delta t}{\Delta y}\left(G_{j, k+1}^{n}-G_{j, k-1}^{n}\right) .
$$

The linear initial-value problem stability condition for this algorithm is

$$
\Delta t \leqslant \frac{1}{\left[\frac{\rho(A)}{\Delta x}+\frac{\rho(B)}{\Delta y}\right]}
$$

where $\rho(A)$ and $\rho(B)$ are, respectively, the spectral radii of the Jacobians $A=\partial F / \partial u$ and $B=\partial G / \partial u$, and it has been assumed that $A$ and $B$ are simultaneously symmetrizable.

In [1] it has been shown that the stability condition (1.3) can be substantially improved by using a Modified Leap-Frog (M.L.-F.) scheme

$$
u_{j, k}^{n+1}=u_{j, k}^{n-1}+\frac{\Delta t}{\Delta x}\left(\widetilde{F}_{j+1, k}^{n}-\widetilde{F}_{j-1, k}^{n}\right)+\frac{\Delta t}{\Delta y}\left(\widetilde{G}_{j, k+1}^{n}-\widetilde{G}_{j, k-1}^{n}\right)
$$

Received March 6, 1978

AMS (MOS) subject classifications (1970). Primary 65M10.

* This research has been sponsored in part by the Air Force Office of Scientific Research (NAM) through the European Office of Aerospace Research, AFSC, United States Air Force, under Grant AFOSR 76-3035.

**This paper was prepared as a result of work performed under NASA Contract Number NAS1-14101 while the second author was in residence at ICASE, NASA Langley Research Center, Hampton, Virginia 23665. 
where

$$
\widetilde{F}_{j, k}^{n}=F\left(1 / 2\left(u_{j, k+1}+u_{j, k-1}\right)\right)
$$

and

$$
\widetilde{G}_{j, k}^{n}=G\left(1 / 2\left(u_{j+1, k}+u_{j-1, k}\right)\right) .
$$

Not only does (1.4) have the better stability condition

$$
\Delta t \leqslant \min \left\{\frac{\Delta x}{\rho(A)} ; \frac{\Delta y}{\rho(B)}\right\}
$$

but it also has a smaller phase error than the standard L.-F. scheme, (1.2).

In this work we intend to present several stable ways of treating the boundary conditions for the Modified Leap-Frog (M.L.-F.) scheme. In deriving these boundary conditions it was found that there is also a need to explore what kind of boundary treatments are stable for the standard two-dimensional L.-F. scheme (1.2).

2. Stability Results. In this section we consider the equation

$$
u_{t}=u_{x}+u_{y}, \quad 0 \leqslant x<\infty,-\infty<y<\infty .
$$

This equation, with suitable stretching of space coordinates, models the system

$$
u_{t}=A u_{x}+B u_{y}=F_{x}+G_{y},
$$

when $A$ and $B$ can be diagonalized by the same similarity transformation. It has been shown by Kreiss that the initial boundary value problem for (2.2) (even if $A$ and $B$ do not commute) can be analyzed by means of Fourier transforming in one direction. We will follow this method of analysis in order to determine stable treatment at the boundaries.

Gustafsson, Kreiss and Sundström [2] considered the one-dimensional case, namely

$$
u_{t}=A u_{x}=F_{x}, \quad 0 \leqslant x<\infty,
$$

where $A$ is a positive diagonal matrix. They have shown that determination of the boundary values by space extrapolation on the same time level is an unstable procedure. They did present three stable "boundary treatment" schemes (at $x=0$ ):

(i) $u_{0}^{n+1}=2 u_{1}^{n}-u_{2}^{n-1}$,

(ii) $u_{0}^{n+1}=u_{0}^{n}+(\Delta t / \Delta x) A\left(u_{1}^{n}-u_{0}^{n}\right)$,

(iii) $u_{0}^{n+1}+u_{1}^{n+1}-(\Delta t / \Delta x) A\left(u_{1}^{n+1}-u_{0}^{n+1}\right)=u_{0}^{n}+u_{1}^{n}-(\Delta t / \Delta x) A\left(u_{1}^{n}-u_{0}^{n}\right)$.

All three leave the pure initial-value stability condition unaltered,

$$
\Delta t \leqslant \Delta x / \rho(A)
$$

In this paper we will discuss extensions of (i) and (ii) to the two-dimensional case. It is readily seen that such extensions (and generalizations) of the boundary conditions (i) and (ii) are not unique. For example, condition (i) may be extended as

$$
u_{0, k}^{n+1}=2 u_{1, k}^{n}-u_{2, k}^{n-1}
$$


On the other hand, one may view (i) as an extrapolation in the characteristic direction in the $x-t$ plane, and then it is reasonable to extend (i) as an extrapolation along the characteristic in the $x-y-t$ space. This will result in the boundary condition

$$
u_{0, k}^{n+1}=2 u_{1, k+1}^{n}-u_{2, k+2}^{n-1} .
$$

Similarly, the one-dimensional boundary condition (ii) may be generalized to

$$
u_{0, k}^{n+1}=u_{0, k}^{n}+\frac{\Delta t}{\Delta x}\left(F_{1, k}^{n}-F_{0, k}^{n}\right)+\frac{\Delta t}{2 \Delta y}\left(G_{0, k+1}^{n}-G_{0, k-1}^{n}\right)
$$

or, it may be extended to

$$
u_{0, k}^{n+1}=u_{0, k}^{n}+\frac{\Delta t}{\Delta x}\left(\widetilde{F}_{1, k}^{n}-{\widetilde{F_{0, k}}}_{0, k}\right)+\frac{\Delta t}{\Delta y}\left(\widetilde{\widetilde{G}}_{0, k+1}^{n}-\widetilde{G}_{0, k}^{n}\right),
$$

where the $\widetilde{\widetilde{F}}$ 's and the $\widetilde{G}$ 's are defined by

$$
\widetilde{F}_{1, k}=F\left(1 / 2\left(u_{1, k+1}+u_{1, k}\right)\right), \quad \widetilde{\widetilde{G}}_{0, k+1}=G\left(1 / 2\left(u_{1, k+1}+u_{0, k+1}\right)\right) .
$$

As far as condition (iii) is concerned, we feel that it is much harder to apply it in the two-dimensional case since it involves the inversion of a matrix in the linear case and the solution of nonlinear algebraic equations, otherwise. Therefore, we shall not consider the extension of (iii) to the two-dimensional case.

At this stage we would like to discuss briefly the way of checking stability. The analysis is based on assuming that the finite-difference equations (1.2) and (1.4) have solution of the type

$$
u_{j, k}^{n}=z^{n} \kappa^{j} e^{i k \eta},
$$

where the indices $n, j, k$ are those appearing in the finite-difference schemes and $i=$ $\sqrt{-1}$. We will look for solutions such that $|z|>1,|\kappa|<1$ in order to establish instability. If we get a solution such that $|z|=1,|\kappa|=1$, we will check the origin of this solution, i.e., how does a perturbation in $z$ affect $\kappa$. Substituting (2.8) into (1.2) and (1.4) gives, respectively, the following characteristic equations:

$$
\kappa\left(z^{2}-1\right)=z\left[\lambda\left(\kappa^{2}-1\right)+2 i \lambda \kappa \sin \eta\right]
$$

and

$$
\kappa\left(z^{2}-1\right)=\lambda z\left[\kappa^{2} e^{i \eta}-e^{-i \eta}\right] .
$$

We assumed that $F=G=u$ and $\lambda=\Delta t / \Delta x=\Delta t / \Delta y$. Within the limitation of linear stability analysis this assumption is not severe since by "stretching" the $x, y$ and $t$ coordinates one may account for different (constant) coefficients in the partial differential equations.

The results of the stability analysis are summarized in the following lemmas:

LEMmA 1. The L.-F. scheme (1.2) with the boundary condition (2.3) is stable under the initial-value stability condition, (1.3). 
Proof. Boundary condition (2.3) leads to the resolvent equation

$$
(z-\kappa)^{2}=0 \text {. }
$$

Equation (2.9) together with (2.11) yields

$$
z^{2}-2 i\left(\frac{\lambda}{1-\lambda} \sin \eta\right) z-1=0
$$

where $-1 \leqslant \sin \eta \leqslant 1$ and, from (1.3), $0<\lambda \leqslant 1 / 2$. Solving for $z$, we get

$$
z=i \gamma \pm \sqrt{1-\gamma^{2}}
$$

where

$$
\gamma=\frac{\lambda}{1-\lambda} \sin \eta, \text { i.e. }-1<\gamma<1 \text {. }
$$

Thus $|z|=1$, and there is no eigenvalue $|z|>1$ for this problem. There remains to check for the possible existence of a generalized eigenvalue. Let $\theta$ be defined by $\gamma=$ $\sin \theta$, and therefore $z=e^{i \theta}$. Assume that (2.11) implies that for some $\theta$ we have $\kappa_{0}$ $=z_{0}$, and let us now check what happens if we perturb $z$ by a small real number, $\epsilon$.

Take $z=z_{0}+\epsilon$; then $\kappa=\kappa_{0}+\delta=z_{0}+\delta$. Substituting these expressions into (2.9) and neglecting terms which are $O\left(\epsilon^{2}\right), O\left(\delta^{2}\right), O(\epsilon \delta)$ and higher, one gets

$$
\frac{\delta}{\epsilon}=\frac{i \beta z_{0}-\lambda-(2-\lambda) z_{0}^{2}}{-i \beta z_{0}-1+(1-2 \lambda) z_{0}^{2}},
$$

where $\beta=2 \lambda \sin \eta$. Using the fact (see Eq. (2.12)) that $i \beta z_{0}=(1-\lambda)\left(z_{0}^{2}-1\right)$, we get from (2.15)

$$
\frac{\delta}{\epsilon}=\frac{1}{\lambda}>0
$$

Thus, for a $z$-value approaching $z_{0}$ from outside the unit circle there corresponds a $k$ approaching $\kappa_{0}$ also from outside the unit circle. We conclude, therefore, that there are no generalized eigenvalues and the scheme is stable.

LEMmA 2. The M.L.-F. scheme (1.4) with the boundary condition (2.3) is unconditionally unstable.

Proof. We have the same resolvent as in Lemma 1, namely

$$
(\kappa-z)^{2}=0,
$$

which upon substitution into (2.10) yields, upon rearranging

$$
z^{2}=\frac{1-\lambda e^{-i \eta}}{1-\lambda e^{i \eta}}
$$

Again we have $|z|=1$, and we have to determine the behavior of $\kappa$ as $z$ approaches the unit disc from the outside. We set $z=z_{0}+\epsilon$ where 


$$
z_{0}=\left(\frac{1-\lambda e^{-i \eta}}{1+\lambda e^{i \eta}}\right)^{1 / 2},
$$

and then $\kappa=\kappa_{0}+\delta=z_{0}+\delta$. We substitute these values of $z$ and $\kappa$ into (2.10) and collect terms in powers of $\epsilon$ and $\delta$ to get

$$
\begin{array}{r}
z_{0}\left[\left(z_{0}^{2}-1\right)-\lambda z_{0}^{2} e^{i \eta}+\lambda e^{-i \eta}\right]+\delta\left(z_{0}^{2}-1-2 \lambda z_{0}^{2} e^{i \eta}\right)+\epsilon\left(2 z_{0}^{2}-\lambda z_{0}^{2} e^{i \eta}+\lambda e^{-i \eta}\right) \\
+O\left(\epsilon^{2}, \delta^{2}, \epsilon \delta\right)=0 .
\end{array}
$$

The expression in the square brackets vanishes by virtue of (2.17); and to first order in $\epsilon$ and $\delta$ we have

$$
\frac{\delta}{\epsilon}=\frac{2 z_{0}^{2}-\lambda z_{0}^{2} e^{i \eta}+\lambda e^{-i \eta}}{1-z_{0}^{2}+2 \lambda z_{0}^{2} e^{i \eta}},
$$

which, upon using (2.17) and rearranging, reduces to

$$
\frac{\delta}{\epsilon}=\frac{1-\lambda \cos \eta}{\lambda(\cos \eta-\lambda)} .
$$

Recall that (with $F=G=u$ ) for the M.L.-F. the initial-value stability condition is $\lambda<1$ rather than $\lambda<1 / 2$ as for the standard L.F. For $0<\lambda<1$ and $-1 \leqslant \cos \eta \leqslant$ 1 we can for each $\lambda$ find $\eta$ such that $\delta / \epsilon<0$. In particular, for $\eta=\pi / 2$ we have $\delta / \epsilon$ $=-1 / \lambda^{2}$; and thus $z=-1$ is a generalized eigenvalue of $(2.11)$ and the boundary condition (2.3) renders the scheme unconditionally unstable.

LEMma 3. The L.-F. scheme (1.2) with the boundary condition (2.4) is unconditionally unstable.

Proof. Using (2.8) the resolvent equation corresponding to (2.4) is

$$
z^{2} e^{-i \eta}=2 \kappa z-\kappa^{2} e^{i \eta}
$$

or

$$
z=\kappa e^{i \eta}
$$

We will demonstrate instability by finding a particular generalized eigenvalue. For $\eta$ $=\pi,(2.19)$ leads to $z=-\kappa$. For this case, the characteristic equation (2.9) becomes

$$
-z\left(z^{2}-1\right)=\lambda z\left(z^{2}-1\right) .
$$

The value $z=-1(\kappa=1)$ is a root of (2.20). Perturbing (2.9) with $z=-1-\epsilon(\epsilon>$ 0 ) and $\kappa=1+\delta$, one gets $\delta=-\epsilon / \lambda$ and, therefore, $|\kappa|<1$. Thus, we have just shown that $\kappa=1, z=-1$ is a generalized eigenvalue.

LEMma 4. The M.L.-F. scheme (1.4) with the boundary condition (2.4) is stable under the initial value stability condition (1.7).

Proof. The resolvent equation is the same as the one obtained in Lemma 3. Substituting (2.19) into the modified characteristic equation, (2.10), yields $z^{2}-1=$ $\lambda\left(z^{2}-1\right)$ and, therefore, $z=z_{0}= \pm 1$ and $\kappa_{0}=z_{0} e^{-i \eta}$. To check for the possible 
existence of a generalized eigenvalue set $z=z_{0}+\epsilon, \epsilon$ real, and $\kappa_{0}=\kappa_{0}+\delta=$ $z_{0} e^{-i \eta}+\delta, \delta$ not necessarily real. Perturbing (2.10) with these quantities and retaining first order terms only in $\epsilon$ and $\delta$ yields the result $\delta=\epsilon e^{-i \eta}$ from which, if $z=$ $z_{0}+\epsilon, \epsilon$ real, then $\kappa=\left(z_{0}+\epsilon\right) e^{-i \eta}$; and hence if $|z|>1$, then $|\kappa|>1$, and there is no generalized eigenvalue.

LEMmA 5. The L.-F. scheme (1.2) with the boundary condition (2.5) is stable under the condition

$$
\lambda=\frac{\Delta t}{\Delta x} \rho(A) \leqslant .4, \quad \frac{\Delta t}{\Delta y} \rho(B) \leqslant .4 .
$$

For $.4<\lambda<.5$ there is a generalized eigenvalue.

Proof. Associated with the boundary condition (2.5) is the resolvent equation (with $u_{j, k}^{n} \sim z^{n} \kappa^{j} e^{i k \eta}$ )

$$
z=1+\lambda(\kappa-1)+i \lambda \sin \eta
$$

Eliminating $z$ between (2.22) and the characteristic equation (2.9), we get a quadratic equation in $k$

$$
\left[\lambda(1-\lambda)-i \lambda^{2} \sin \eta\right] \kappa^{2}+\left[2 \lambda(\lambda-1)+\lambda^{2} \sin \eta\right] \kappa+\lambda(1-\lambda)+i \lambda^{2} \sin \eta=0
$$
with the notation

$$
\alpha=\lambda(1-\lambda) ; \quad \beta=\lambda^{2} \sin \eta ; \quad \gamma=2 \lambda(\lambda-1)+\lambda^{2} \sin ^{2} \eta .
$$

The solution of $(2.23)$ is

$$
\kappa=\frac{-\gamma \pm \sqrt{\gamma^{2}-4\left(\alpha^{2}+\beta^{2}\right)}}{2(\alpha-i \beta)} .
$$

The radical in (2.25) is evaluated using (2.24),

$$
\begin{aligned}
\gamma^{2}-4\left(\alpha^{2}+\beta^{2}\right)= & 4 \lambda^{2}(\lambda-1)^{2}+4 \lambda^{3}(\lambda-1) \sin ^{2} \eta+\lambda^{4} \sin ^{4} \eta \\
& -4 \lambda^{2}(1-\lambda)^{2}-4 \lambda^{4} \sin ^{2} \eta \\
= & \lambda^{3} \sin ^{2} \eta\left(\lambda \sin ^{2} \eta-4\right)<0 ;
\end{aligned}
$$

and therefore,

$$
\kappa=\frac{-\gamma \pm i \sqrt{4\left(\alpha^{2}+\beta^{2}\right)-\gamma^{2}}}{2(\alpha-i \beta)}=X+i Y
$$

where

$$
\begin{aligned}
& X=\frac{1}{2\left(\alpha^{2}+\beta^{2}\right)}\left(-\alpha \gamma \mp \beta \sqrt{4\left(\alpha^{2}+\beta^{2}\right)-\gamma^{2}}\right), \\
& Y=\frac{1}{2\left(\alpha^{2}+\beta^{2}\right)}\left(-\beta \gamma \pm \alpha \sqrt{4\left(\alpha^{2}+\beta^{2}\right)-\gamma^{2}}\right) .
\end{aligned}
$$

From (2.26) it is clear that $|\kappa|=\left(X^{2}+Y^{2}\right)^{1 / 2}=1$. Substituting $\kappa$ from (2.26) into 
the resolvent equation (2.22), we get for $z$

$$
z=1-\lambda+\lambda X+i \lambda(Y+\sin \eta)
$$

Using (2.24), it is easy to show that also $|z|=1$. We now have to check out the possible existence of a generalized eigenvalue. Let $z_{0}=e^{i \psi}$ be given by (2.29) and $\kappa_{0}=$ $e^{i \phi}$ be given by (2.26). Perturb $z_{0}$ and $\kappa_{0}$ by setting $z=(1+\epsilon) e^{i \psi}, \epsilon>0$ and $\kappa=$ $(1+\delta) e^{i \phi}$. Substitution of these perturbed values into the characteristic equation (2.9) yields, to first order in $\epsilon$ and $\delta$,

$$
e^{i \psi}(1+\epsilon)-e^{-i \psi}(1-\epsilon)=\lambda\left[e^{i \psi}(1+\delta)-e^{-i \psi}(1-\delta)\right]+2 i \lambda \sin \eta
$$

from which we get

$$
\frac{\epsilon}{\delta}=\frac{\lambda \cos \phi}{\cos \psi}=\frac{\lambda\left(\text { Real } \kappa_{0}\right)}{\operatorname{Real} z_{0}}
$$

We will have stability (i.e., no generalized eigenvalue) if

$$
\frac{\epsilon}{\delta}=\frac{\lambda \cos \phi}{\cos \psi}>0 .
$$

From (2.29) we have

$$
\cos \psi=\operatorname{Real} z_{0}=1-\lambda+\lambda\left(\operatorname{Real} \kappa_{0}\right)>0
$$

since $0<\lambda<.5$ and $\mid$ Real $\kappa_{0} \mid<1$. Thus the question reduces to deciding under what conditions Real $\kappa_{0}>0$. The requirement of Real $\kappa_{0}>0$ is, from (2.27), equivalent to (since $\gamma<0$; see (2.24))

$$
\alpha^{2} \gamma^{2}>\beta^{2}\left[4\left(\alpha^{2}+\beta^{2}\right)-\gamma^{2}\right] \quad \text { or } \quad \gamma^{2}>4 \beta^{2}
$$

and, since $\gamma<0$,

$$
|\gamma|>2|\beta|
$$

Using from (2.24) the expressions for $\beta$ and $\gamma$, Eq. (2.31) becomes

$$
2 \lambda(1-\lambda)-\lambda^{2} \sin ^{2} \eta>2 \lambda|\sin \eta| .
$$

The most severe requirement on $\lambda$ in (2.32) takes place when $|\sin \eta|=1$, and thus we have $2 \lambda>5 \lambda^{2}$ or

$$
\lambda<0.4
$$

This is sufficient for stability. To show that (2.33) is also a necessary condition we show that for $\lambda>.4$ we can find a generalized eigenvalue. Take $\eta=\pi / 2$, and we find that for $\lambda>.4, X=$ Real $\kappa_{0}<0$; and hence (2.30) is violated. This completes the proof.

As far as we are aware, this is the first known example that the boundary treatment modifies the stability condition rather than negating or leaving it unaltered. 
LEMma 6. The M.L.-F. scheme (1.4) with the boundary condition (2.5) is unconditionally unstable.

Proof. We give a counterexample to stability. Take $\eta=\pi$ and the appropriate characteristic and resolvent equations, respectively, (2.10) and (2.22) take the form

$$
\kappa\left(z^{2}-1\right)=-\lambda z\left(\kappa^{2}-1\right)
$$

and

$$
z-1=\lambda(\kappa-1)
$$

A solution of (2.34) and (2.35) is $z_{0}=\kappa_{0}=1$. Perturb by taking $z=1+\epsilon$ and $\kappa=1+\delta$. To first order in $\epsilon$ and $\delta$ Eq. (2.34) becomes

$$
\epsilon=-\lambda \delta
$$

i.e., $z=\kappa=1$ is a generalized eigenvalue and we have instability.

LEMMA 7. The L.-F. scheme (1.2) with boundary condition (2.6) is unconditionally unstable.

Proof. The proof is similar to that of Lemma 6. The resolvent equation associated with (2.6) is

$$
z=1+\lambda\left(\kappa e^{i \eta}-1\right)
$$

Substitute $\eta=\pi$ into the characteristic equation (2.9) and (2.37) to get

$$
\kappa\left(z^{2}-1\right)=\lambda z\left(\kappa^{2}-1\right)
$$

and

$$
z-1=-\lambda(\kappa+1)
$$

which are solved by $z_{0}=1$ and $\kappa_{0}=-1$. Perturb by setting $z=1+\epsilon$ and $\kappa=$ $-(1+\delta)$. To first order in $\epsilon$ and $\delta$ Eq. (2.38) becomes $\epsilon=-\lambda \delta$, i.e., $z=1, \kappa=-1$ is a generalized eigenvalue and we have instability.

LEMMA 8. The M.L.-F. scheme (1.4) with boundary condition (2.6) is stable under the same stability condition as the initial-value problem.

Proof. Substituting $\kappa_{1} \doteq \kappa e^{i \eta}$ in the appropriate characteristic equations and resolvent (2.10) and (2.37), we get, respectively:

$$
\kappa_{1}\left(z^{2}-1\right)=\lambda z\left(\kappa_{1}^{2}-1\right)
$$

and

$$
z=1+\lambda\left(\kappa_{1}-1\right)
$$

The set (2.40), (2.41) models the case of one-dimensional L.-F. with one-sided differencing at the boundary (see boundary condition (ii) on p. 1146). This case was discussed by Gustafsson et al. [2] and shown to be stable. This completes the proof. 
3. Numerical Results. In order to verify the analyses of Section 1 we solved numerically the test problem

$$
\begin{gathered}
u_{t}=u_{x}+u_{y}, \quad 0 \leqslant x<\infty, 0 \leqslant y<\infty, \\
u(x, y, 0)=\sin 2 \pi x \sin 2 \pi y,
\end{gathered}
$$

the solution to which is

$$
u(x, y, t)=\sin 2 \pi(x+t) \sin 2 \pi(y+t)
$$

The numerical solution was carried on the square $0 \leqslant x \leqslant 1,0 \leqslant y \leqslant 1$. Since the initial condition is periodic in $y$ with period 1 , and since the analysis assumed Fourier transformation in the $y$ direction, we have to give periodic conditions on the $y=$ constant boundaries. The characteristics of the transformed equation have negative slope in the $x-t$ plane; and hence, we must specify a solution on $x=1$ and a boundary condition at $x=0$. The various conditions were, then, chosen as follows:

$$
u(1, y, t)=\sin 2 \pi t \sin 2 \pi(y+t) .
$$

\begin{tabular}{|c|c|c|c|c|c|c|c|c|c|}
\hline \multirow{2}{*}{\multicolumn{2}{|c|}{ Method }} & \multicolumn{2}{|c|}{$\begin{array}{c}\text { Boundary condition } \\
\text { Eq. }(2.3)\end{array}$} & \multicolumn{2}{|c|}{$\begin{array}{c}\text { Boundary condition } \\
\text { Eq. }(2.4)\end{array}$} & \multicolumn{2}{|c|}{$\begin{array}{c}\text { Boundary condition } \\
\text { Eq. }(2.5)\end{array}$} & \multicolumn{2}{|c|}{$\begin{array}{c}\text { Boundary condition } \\
\text { Eq. }(2.6)\end{array}$} \\
\hline & & $\begin{array}{l}\text { Max } \\
\text { error }\end{array}$ & $\begin{array}{l}\text { Relative } \\
\left\|L_{2}\right\| \text { error }\end{array}$ & $\begin{array}{l}\text { Max } \\
\text { error }\end{array}$ & $\begin{array}{c}\text { Relative } \\
\left\|L_{2}\right\| \text { error }\end{array}$ & $\begin{array}{l}\text { Max } \\
\text { error }\end{array}$ & $\begin{array}{l}\text { Relative } \\
\left\|L_{2}\right\| \text { error }\end{array}$ & $\begin{array}{l}\text { Max } \\
\text { error }\end{array}$ & $\begin{array}{l}\text { Relative } \\
\left\|L_{2}\right\| \text { error }\end{array}$ \\
\hline L. $\cdot \mathrm{F}$ & $\lambda=.39$ & .019 & .0088 & \multicolumn{2}{|r|}{ unstable } & .017 & .0099 & \multicolumn{2}{|c|}{ unstable } \\
\hline L. $F$ & $\lambda=.41$ & .0165 & .0080 & \multicolumn{2}{|r|}{ unstable } & .015 & .0093 & \multicolumn{2}{|c|}{ unstable } \\
\hline L..$F$ & $\lambda=.47$ & .0089 & .0060 & \multicolumn{2}{|r|}{ unstable } & \multicolumn{2}{|c|}{ unstable } & \multicolumn{2}{|c|}{ unstable } \\
\hline L. $\cdot \mathrm{F}$ & $\lambda=.49$ & .0058 & .0078 & \multicolumn{2}{|r|}{ unstable } & & stable & \multicolumn{2}{|c|}{ unstable } \\
\hline \multicolumn{2}{|c|}{ M.L.-F $\lambda=.9$} & \multicolumn{2}{|c|}{ unstable } & .0205 & .0171 & \multicolumn{2}{|c|}{ unstable } & .0222 & .0173 \\
\hline \multicolumn{2}{|c|}{ M.L.F $\lambda=.99$} & \multicolumn{2}{|c|}{ unstable } & & .0017 & \multicolumn{2}{|c|}{ unstable } & .0023 & .0017 \\
\hline
\end{tabular}

We used both the Leap-Frog and the Modified Leap-Frog schemes, using at $x=0$ the various boundary conditions considered in the previous section. The runs were of 1000 time steps. The results are summarized in the following table:

The tabulated values amply verify the theoretical results.

We used for the L.F. calculation the known solution that is

$$
u(x, 0, t)=u(x, 1, t)=\sin 2(x+t) \sin 2 t .
$$

The results for the M.L.-F. are for the periodic condition $u(x, 0, t)=u(x, 1, t)$.

It is interesting to note that if, instead of (3.5), we used the periodicity condition $u(x, 0, t)=u(x, 1, t)$, then the :lumerical computations failed to show the instability for $\lambda>.4$ in the case of the L.-F. with boundary condition (2.5). This is due, we think, to the fact that the dissipation introduced by the extrapolation overcomes the weak instability due to the existence of a generalized eigenvalue for $\lambda>4$. 
4. Analysis of a Two-Dimensional System. In this section, we would like to explore the stability of the Leap-Frog scheme applied to the two-dimensional hyperbolic system

$$
\frac{\partial}{\partial t}\left(\begin{array}{l}
u \\
v
\end{array}\right)=\left(\begin{array}{cc}
1 & 0 \\
0 & -1
\end{array}\right) \frac{\partial}{\partial x}\left(\begin{array}{l}
u \\
v
\end{array}\right)+\left(\begin{array}{ll}
0 & 1 \\
1 & 0
\end{array}\right) \frac{\partial}{\partial y}\left(\begin{array}{l}
u \\
v
\end{array}\right)
$$

in the half-plane $0 \leqslant x<\infty,-\infty<y<\infty(t \geqslant 0)$ with the boundary condition

$$
v(0, y, t)=0 \text {. }
$$

Since only one characteristic is positive, $u$ cannot be specified at $x=0$ (see [3]) and will be determined by the extrapolation (2.3).

We set in (4.1)

$$
u_{j, k}^{n}=\alpha z^{n} \kappa^{j} e^{i k \eta}, \quad v_{j, k}^{n}=\beta z^{n} \kappa^{j} e^{i k \eta}
$$

to get

$$
\begin{aligned}
& {\left[\kappa\left(z^{2}-1\right)-\lambda z\left(\kappa^{2}-1\right)\right] \alpha+[2 i \lambda \kappa z \sin \eta] \beta=0,} \\
& {[2 i \lambda \kappa z \sin \eta] \alpha+\left[\kappa\left(z^{2}-1\right)+\lambda z\left(\kappa^{2}-1\right)\right] \beta=0 .}
\end{aligned}
$$

A solution for $\alpha$ and $\beta$ exists if the coefficient matrix vanishes. This requirement leads to the following characteristic equation:

$$
\lambda^{2} z^{2} \kappa^{4}-\left[\left(z^{2}-1\right)^{2}+2 \lambda^{2} z^{2}+4 \lambda^{2} z^{2} \sin ^{2} \eta\right] \kappa^{2}+\lambda^{2} z^{2}=0 .
$$

Let $\kappa^{2}=\mu$. It is clear that there are two solutions $\mu_{1}$ and $\mu_{2}$ such that

$$
\mu_{1} \mu_{2}=1 \text {, }
$$

say $\left|\mu_{1}\right| \leqslant 1$ and $\left|\mu_{2}\right| \geqslant 1$.

For every $|z|>1$ we are interested only in the two solutions $\kappa_{1}=\sqrt{\mu_{1}}$ and $\kappa_{2}=-\sqrt{\mu_{1}}$ since with $\kappa_{3,4}= \pm \sqrt{\mu_{2}}$ the solution will not be in $L_{2}$. Note that $\kappa_{1}=$ $-\kappa_{2}$ and that $\left|\kappa_{1}\right|=\left|\kappa_{2}\right|<1$.

Having found that we need only two of the four $\kappa$ 's, the solution must be of the form

$$
\begin{aligned}
& u_{j, k}^{n}=z^{n} e^{i k \eta}\left(\alpha \kappa_{1}^{j}+\gamma \kappa_{2}^{j}\right), \\
& v_{j, k}^{n}=z^{n} e^{i k \eta}\left(\beta \kappa_{1}^{j}+\delta \kappa_{2}^{j}\right) .
\end{aligned}
$$

With these $u_{j, k}^{n}$ and $v_{j, k}^{n}$ Eqs. (4.4) and (4.5) are valid for $\kappa=\kappa_{1}$ and also for $\kappa=\kappa_{2}$ if we replace $\alpha$ and $\beta$ by $\gamma$ and $\delta$, respectively. We then can write, using (4.4),

$$
\begin{aligned}
& \frac{\alpha}{\beta}=-\frac{2 i \lambda z \kappa_{1} \sin \eta}{\left(z^{2}-1\right) \kappa_{1}-\lambda z\left(\kappa_{1}^{2}-1\right)}, \\
& \frac{\gamma}{\delta}=-\frac{2 i \lambda z \kappa_{2} \sin \eta}{\left(z^{2}-1\right) \kappa_{2}-\lambda z\left(\kappa_{2}^{2}-1\right)}=-\frac{2 i \lambda z \kappa_{1} \sin \eta}{\left(z^{2}-1\right) \kappa_{1}+\lambda z\left(\kappa_{1}^{2}-1\right)} .
\end{aligned}
$$


In view of (4.9) the boundary condition (4.2) becomes $\beta+\delta=0$, or

$$
\beta=-\delta,
$$

while the extrapolation (2.3) yields

$$
\alpha\left(\kappa_{1}-z\right)^{2}+\gamma\left(\kappa_{2}-z\right)^{2}=0,
$$

which is equivalent to (since $\kappa_{1}=-\kappa_{2}$ )

$$
\alpha\left(\kappa_{1}-z\right)^{2}+\gamma\left(\kappa_{1}+z\right)^{2}=0 .
$$

Eliminating $\alpha, \beta, \gamma$ and $\delta$ from (4.10) to (4.13), we get

$$
\lambda^{2} z^{2} \kappa_{1}^{4}+\lambda(\lambda-2)\left(z^{2}-1\right) z^{2} \kappa_{1}^{2}-\lambda^{2} z^{4}=0 .
$$

If we now rewrite (4.6) with $\kappa=\kappa_{1}$ and eliminate $\kappa_{1}$ between (4.14) and (4.6), we get the following eighth-order algebraic equation for $z$ :

$$
a_{8} z^{8}-a_{6} z^{6}+a_{4} z^{4}-a_{2} z^{2}+a_{0}=0
$$

where

$$
\begin{aligned}
& a_{8}=a_{0}=(1-\lambda)^{2}, \\
& a_{6}=a_{2}=4\left(1-\lambda^{2} \theta\right)+\lambda^{2}(2-\lambda)^{2}+\lambda^{4}+2 \lambda(\lambda-2)\left(2-\lambda^{2} \theta\right), \\
& a_{4}=6-8 \lambda^{2} \theta+4 \lambda^{4} \theta^{2}+2 \lambda^{2}(2-\lambda)^{2}-2 \lambda^{4}+\lambda(\lambda-2)\left(6-4 \lambda^{2} \theta\right), \\
& \theta=1+2 \sin ^{2} \eta .
\end{aligned}
$$

We made a (numerical) parametric study of (4.15) with $0<\lambda \leqslant 1 / 2,1 \leqslant \theta \leqslant 3$ and for each $z,|z|>1$, so found we computed $\kappa_{1}$ from (4.14). We found no solution of (4.15) with $|z|>1$ such that the corresponding $\kappa_{1}$ computed from (4.14) was smaller than 1 in magnitude. This shows stability. A similar analysis shows that the M.L.-F. for (4.1) with boundary scheme (2.4) is stable.

\footnotetext{
Department of Mathematical Sciences Division of Applied Mathematics

Tel-Aviv University

Tel-Aviv, Israel

ICASE

NASA Langley Research Center

Hampton, Virginia 23665
}

1. S. ABARBANEL \& D. GOTTLIEB, "A note on the leap-frog scheme in two and three dimensions," J. Computational Phys., v. 21 (3), 1976, pp. 351-355.

2. B. GUSTAFSSON, H.-O. KREISS, \& A. SUNDSTRÖM, "Stability theory of difference approximations for mixed initial boundary value problems. II," Math. Comp., v. 26, 1972, pp. $649-686$.

3. H. O. KREISS \& J. OLIGER, Methods for the Approximate Solution of Time Dependent Problems, GARP Publications Series, No. 10, 1973.

4. K. W. MORTON, "Initial-value problems by finite difference and other methods," The State of the Art in Numerical Analysis (Proc. Conf., Univ. York, Heslington, 1976), Academic Press, London, 1977, pp. 699-756. 